\title{
PHYSICAL METHODS FOR THE IDENTIFICATION OF MATERIALS
}

$\mathrm{T}$

THE X-ray Analysis Group of the Institute of Physics held a joint meeting with the Midland Branch of the Institute on October 4 in Birmingham, to discuss "Physical Methods for the Identification of Materials". Sir Lawrence Bragg gave a historical summary of different methods which had been used for the identification of materials, and Prof. M. L. E. Oliphant commented on the importance of the subject in nuclear physics. A knowledge of the corrosion products of metals, for example, involves physical methods of identification.

The first paper, by Dr. M. W. Porter and Mr. R. C. Spiller, on the Barker Index ${ }^{1}$, dealt with the development of morphological methods. The authors described the development by T. V. Barker of a simplified systematic crystallography which ensures that the surface of a crystal can be (1) unambiguously described in terms of a standard set of axes and parameters derivable from the form development, (2) classified morphologically according to the angular values, (3) identified on any future occasion by the simple process of measurement, geometrical analysis and reference to tables. Precise rules and principles are laid down which transform descriptive crystallography into a determinative science ${ }^{2}$. A crystal is measured in the usual way on a single-circle or twocircle goniometer, a stereographic or gnomonic projection is made and the symmetry is ascertained.

The authors then described how the principle of simple indices is applied to find an unambiguous set of axes and parameters (setting). A correct application of the rules leads independent workers to choose identical settings for crystals of the same substance. Certain angles are then chosen as classification angles and are entered in their proper place in the Barker Index by means of the main classifying angle. Tests have shown that the identification of unknown crystals is then possible. Small crystals generally provide the best reflecting faces for accurate measurement. The ideal size is about $5 \mathrm{~mm} . \times 3 \mathrm{~mm} . \times 3 \mathrm{~mm}$., but crystals as small as $1 / 10 \mathrm{~mm}$. across can be measured and identifiod ${ }^{3}$. Two-circle goniometry has the great advantage that small and fragile crystals need only be mounted once or twice for identification.

The present position of the Index is that all the tetragonal and orthorhombic compounds in Groth's "Chemische Krystallographie" have been completed, and crystal descriptions published since Groth are being included. The entries for some of the trigonal, hexagonal and monoclinic and all the anorthic compounds require checking to ensure the greatest possible standard of accuracy. Discussions are now taking place as to the best method of publishing the Index.

In commenting on the Barker method, Dr. M. H. Hey said that it has been overshadowed by the contemporaneous development of X-ray methods, but has its own appropriate field of use. It is better suited to organic compounds, which show few isomorphous series, than to inorganic. It is the only method capable of identifying the original material of pseudomorphs or crystal casts and is quite unaffected by inclusions, however large their amount. It is applicable to metamict materials which show enough external forms. But it fails entirely with fragmentary material, aggregates, felts, spherulites, or other forms lacking crystal faces, and with small crystals having matt or highly curved faces.
The second paper, on optical methods, was given by Dr. N. F. M. Henry. Crystal optics, he said, was developed mainly by mineralogists and petrologists ; but chemists are, to an increasing extent, using optical methods in the study both of organic and inorganic substances. These methods are among the most useful in identification work and the application of the polarizing microscope should be the first step, followed by qualitative chemical tests and powder photographs if necessary. In most cases one is working within a limited range of possibilities, and optical methods are often sufficient in themselves, while their rapidity is a great advantage. They are not applicable to extremely fine-grained specimens smaller than $0.001 \mathrm{~mm}$., in which case powder photographs have to be used; but they can be readily used for organic substances, and in this field powder photography is less reliable in general than in the field of inorganic substances.

Measurement of refractive indices is the chief optical method of identification. When a crystal has been immersed in an oil of known refractive index, one or both of two techniques can be used to compare its index with that of the oil. Of these, the Becke line technique is the most sensitive (accurate to \pm 0.002 ) and the more generally useful. The Van der Kolk method is more valuable for crystals and fragments with sloping edges. Other optical properties can usually be recorded as well as the refractive indices and are sometimes indispensable for complete identification. Thus a member of the plagioclase series of felspars can be identified by refractive indices alone, whereas the different forms of asbestos can be suitably identified only by the measurement of extinction angles as well as refractive indices. For these and other simple solid-solution series which have been well studied it is often possible to give an estimate of the chemical composition.

The usefulness of the optical method depends, as with other methods, on the existence of full and accurate data. The latest lists contain some 1,400 minerals, more than 2,000 artificial inorganic substances and about 2,700 organic substances.

Mr. F. A. Bannister opened the afternoon session with a paper on single erystal and powder methods. Of the X-ray methods developed for the analysis of crystal structures, the powder method had predominated and there had been a tendency to neglect single-crystal techniques. This is unavoidable when the crystal size is much less than $0.1 \mathrm{~mm}$., but there are good reasons for using single crystals for identification when they are large enough to be isolated and mounted for a rotation photograph : (1) smaller amounts of a substance can be identified by this than by the powder method ; (2) a two-dimensional array of spots on a rotation photograph is a more certain identification than a set of powder lines and can generally be indexed more readily ; $(3)$ the deliberate powdering of soft materials like the serpentines, chlorites and many organic substances for identification is not to be recommended, since many of the powder lines obtained will be poorer in definition than the fibre or plate-aggregate photographs which these materials yield in their pristine condition.

Photographs of crystals set up with a principal axis exactly coincident with the axis of rotation are not the only ones which can be used for identification. Rotation or oscillation photographs of unoriented single crystals can often be identified by using a Bernal $\theta x$ chart or by superposition upon a powder photograph of the same substance. This assumes the 
use of the same radiation, camera diameter, pin-hole collimation of the incident X-ray beam and a broader film than is normally used for powder photographs. The method is especially useful for substances with low unit-cell dimensions such as diamond ${ }^{5}$ and has been used recently for identifying single-crystal grains of magnetite, goethite and hæmatite in fine-grained bauxites. Single-crystal photographs of a mixture of anhedral grains can be employed to separate each constituent phase for chemical analysis. The value of the method in the determinative mineralogy of the opaque metallic sulphides, arsenides, antimonides and tellurides cannot be exaggerated ${ }^{\text {. }}$.

Mr. Bannister then described the development of indexes for identification of materials by the powder method. Even when provided with some preliminary optical and chemical data for an unidentified substance, the number of powder photographs of known materials with which comparison is desirable can be large, so that hunting down the right pattern can be time-consuming. System has been introduced into powder determinations by arranging the spacings and intensities of the powder lines of each substance in the form of a card index. The Americans have chosen to index the strongest three lines of each patterm and list the complete powder data on the first of the three cards, that is, the one with the longest spacing. An account of the American index and British cooperation was given by Dr. A. J. C. Wilson at the annual meeting of the X-ray Analysis Group of the Institute of Physics in July 1945?. Dr. Wilson has collected, edited and sent to Philadelphia a very large part of the first appendix to the original index ${ }^{8}$. The total number of substances indexed is more than 2,500. This includes more than 700 minerals, nearly 150 organic compounds (excluding acetates, oxalates, etc.), and the rest are inorganic substances including metals and alloys. At a conservative estimate the number of mineral entries has to be doubled and the inorganic field greatly extended. The farthest from completion is the organic section.

It is always desirable to confirm an identification suggested by the powder index with direct comparison of the photograph with a photograph of the substance in question. The interchange of microfilm copies of the powder photographs taken in various laboratories is suggested. The index can often be used to identify three or four components of a mixture. Sometimes a single line will evade recognition when all the rest can be tied down to known com. ponents. It is then that one sees the eventual necessity of assigning indices to all the spacings tabulated for a given substance. Otherwise it is impossible to decide whether a weak line additional to those given in the index is due to one of the identified components or to admixture with another substance.

Mr. C. W. Bunn followed with an account of problems of identification in an industrial chemical laboratory. He stated that of the three methods already discussed-morphological, optical and $\mathrm{X}$-ray -only the last two had been used in the laboratory of the Alkali Division of Imperial Chemical Industries, Ltd. Crystals of most specimens submitted are either much too small or not sufficiently well-formed to handle individually by the morphological method. The measurement of refractive indices by the immersion method is widely used, and for direct identification a card index is kept with substances arranged in order of the principal refractive index $\beta$. Refractive indices of particles down to $0.001 \mathrm{~mm}$. can be measured, but accuracy falls off with decrease in size of the crystals. Auxiliary evidence of identification is provided by crystal shape and the microscopic determination of the mutual orientation of the principal optical and the morphological directions.

For identification by X-ray diffraction the powder method has been most used. Mr. Bunn said that he uses a $9-\mathrm{cm}$. diameter camera for most purposes and has been mainly concerned with inorganic substances. The identification of a single substance is usually straightforward, though there are a few cases of different substances having diffraction patterns so closely similar that they might be confused. The constituents of mixtures can often be identified by successive elimination of sets of lines in the pattern; as many as four different crystalline species have been identified in this way'. This is usually only possible if they are all present in proportion greater than 10 per cent. Often, after the elimination process, a few faint lines are left which may give suggestions but are not enough to establish identity; here, chemical and physical methods of concentrating constituents may sometimes be employed. For complex mixtures, it is desirable to use a large-radius camera to attain greater resolution; but it may not be possible to get the full benefit from this practice at present, because recorded data are often not sufficiently accurate. For quantitative analyses (two constituents), a powder photograph of the mixture is compared with standard photographs of mixtures of known proportions of the constituents; for rutileanatase mixtures an answer can be given to $\pm 2 \frac{1}{2}$ per cent by visual inspection. For rapid work with familiar mixtures, a small camera $\left(4 \frac{1}{2} \mathrm{~cm}\right.$. in diameter) has been used to obtain photographs in fifteen minutes; this is for plant control, where early information of the composition of a mixture enabled adjustments to be made.

With regard to the powder photograph index, the American Society for Testing Materials (A.S.T.M.) index is somewhat unwieldy, with its three cards for each substance. A substance can usually be identified by its strongest line, and $\mathrm{Mr}$. Bunn recommends taking all the 'first' cards out to form a more manageable index, keeping the rest as a reserve. His own index is simpler still : on one card, all substances having their strongest line at a particular spacing (to 0.01 A.) are listed, with references to the complete pattern.

The 'strongest line' procedure, or even the use of the strongest three lines, may not always be successful. Mr. Bunn has sometimes been led to the identification of a constituent by lines at small angles which are not the strongest or even among the first three for the substance in question. They are seen because they are in the region of the photograph where there are no other lines, or very few. For this reason there may be a case for making a supplementary index of 'innermost lines'.

Mr. Bunn concluded with a discussion of the scope of the different crystallographic methods of identification. Of these the X-ray method is by far the most powerful and certain, and in his labnratory there is a tendency to use it straight away. There is now a danger that the microscope may be neglected; this would be a mistake, for where the X-ray method is weakest (in the identification of small proportions of constituents) the microscope method is sometimes helpful. As regards the compilation of data, that must wait on experimental work. When card indexes of optical properties or X-ray powder patterns are 
made, the organic substances should be kept separate from the inorganic substances; and further subdivisions may be desirable, so that indexes do not become unwieldy.

Dr. M. Mathieu, of the Ecole de Physique et Chemie, Paris, during a description of studies proceeding in his laboratory, stated that oriented flakes of montmorillonite can contain many 'phases' each with a different water content. Powder photographs, however, would reveal only one simple set of lines. This is characteristic of materials with layer structures and, in particular, clay minerals.

Mr. H. P. Rooksby directed attention to the recent publication of Frevel on the large number of substances in the American Society for Testing Materials index which had as one of the three strong reference lines a spacing between $2 \cdot 5$ and $3 \mathrm{~A} .^{10}$ This disadvantage could be removed by a preliminary spectroscopic analysis and a regrouping of the spacing cards under chemical elements. He also pointed out a difficulty which was well illustrated in identifying the components of a refractory containing silica as the major constituent. What the American index would identify as predominantly mullite, a crystalline aluminium silicate, might also contain up to 40 per cent silica-rich glass not revealed by powder photography.

,Mrs. Barbara Rogers (Department of Chemical Crystallography, Oxford) said that in the identification of organic compounds they had used singlecrystal methods wherever possible, but sometimes, particularly where the preparations were obtained from natural products in small quantities - frequently impure-only powder methods were suitable. In almost all cases they had proved exact enough to discriminate unambiguously between different compounds, even those of essentially similar chemical structure which differ only slightly from each other, as, for example, in the nature of the side chain. Often slight changes in chemical structure are sufficient to produce definite changes in crystal structure and consequently in the appearance of the powder diagrams; even where the two components are practically isomorphous, change in the intensity of the lines can frequently be observed. In certain cases both powder photographs and single-crystal photographs of racemates have proved very similar to those of the optically active components, sug. gesting that a preliminary examination of synthetic inactive products can often usefully be made before resolution into $d$ - and $l$-forms is attempted.

Mr. H. J. Goldschmidt stated that in favourable cases as much as 20 per cent of a second phase might be present in addition to the identified component and yet elude detection by the powder method. $\mathrm{He}_{\theta}$ had found that by interrupting a rotation photograph and keeping the sample stationary for the latter half of the exposure, weak lines, due to an additional phase that might otherwise have been missed, were recorded. In this way a trace of ferrite in austenite had been successfully detected. He suggested that, complementary to the American Society for Testing Materials index, a collection of standard minerals and allied synthetic compounds should be prepared and distributed when required by a central organisation.

Dr. K. L. Goodall foreshadowed an important application of the X-ray powder method to the field of industrial health. Fine air-borne dust responsible for silicosis contains constituents which are not yet satisfactorily determined by standard chemical or optical methods. The X-ray technique may well yield quantitative analyses of the small samples of industrial dust being collected by electrostatic precipitation for this study.

F. A. BanNister.

${ }^{1}$ Nature, 144, 298 (1939).

2 Donnay, J. D. H., Amer. Min., 28, 313 (1943).

${ }^{8}$ Bannister, F. A., and Hey, M. H., Discovery Reports, 13, 60 (1936).

- Bannister, F. A., and Hey, M. H., Mineral. Mag., 24, 49 (1935).

${ }^{5}$ Bannister, F. A., and Lonsdale, K., Mineral. Mag., 26, 315 (1943).

${ }^{6}$ Bannister, F. A., and Hey, M. H., Mineral. Mag., 23, 188 (1932).

J. Sci. Instruments, 22, 131 (1945).

${ }^{8}$ Nature, 155,648 (1945).

- Clark, L. M., and Bunn, C. W., Soc. Chem. Ind., 59, 155 (1940).

${ }^{10}$ Frevel, L. K., Ind. and Eng. Chem., Anal. Ed., 16, 209 (1944).

\section{MITE-TYPHUS AND THE NEW MITE-TYPHUS VACCINE}

$M$ UCH interest has been aroused by the recent release of information about the new vaccine for the protection of man against mite-typhus (scrub. typhus). Mite-typhus has been-and still is-one of the major health risks in the Far East. The name scrub-typhus, which is commonly given to this disease, is somewhat misleading. It was adopted because the disease is prevalent in areas covered with scrub vegetation, which provides a favourable habitat for the larval mite carrying the disease; but experience during the War showed that the disease is just as prevalent, if not more so, in damp areas covered with the very tall tropical kunai grass (see J. B. Logue, U.S. Naval Med. Bull., 43, 645; 1944), which may grow to a height of 10-20 ft., and also in areas where coco-nut palms and other vegetation occur. Damp and swampy country favours the disease. It is found in Japanese river valleys near the sea and for this reason has been called 'Japanese river fever'.

It is clear, therefore, that there is no essential connexion between the disease and scrub vegetation. The essential epidemiological elements are the Rickettsia ( $R$. orientalis) which causes the disease, and the larval mite which transmits this Rickettsia to man from small animals, chiefly rodents. The Japanese call the disease tsutsugamushi (small-insect) disease. Dutch workers in the Netherlands East Indies (see Kouwehaar and Wolff, Trop. Dis. Bull., 33, 442; 1936; and van der Schroeff, ibid., 38, 684; 1941 ) and some other workers (see C. E. Cook, Med. J. Australia, 2, 539 ; 1944) consider that larval ticks may also transmit the disease.

Two valuable accounts of mite-typhus have been published, by Sir John Megaw (Brit. Med. J., 109, July 28, 1945) and in.a leading article in the Lancet (310, March 15, 1945). The Tropical Diseases Bulletin and the Bulletin of War Medicine also contain abstracts of papers by medical men who have had to deal with outbreaks of mite-typhus in the Far East during the War. Experience of the disease gained there has added considerably to our knowledge of it, for mite-typhus is distributed pretty well all over the Far Eastern theatre of operations, and may be expected to occur also in India and Ceylon. It was known to the Chinese so early as the third century A.D. and was attributed by them to the bite of a small red 'insect'.

Another sound reason for calling this disease mitetyphus and not scrub-typhus is the fact that the name mite-typhus helps to differentiate mite-typhus from the other typhus-like diseases which are also 